Article

\title{
Community Structure, Biodiversity, and Ecosystem Services in Treeline Whitebark Pine Communities: Potential Impacts from a Non-Native Pathogen
}

\author{
Diana F. Tomback ${ }^{1, *}$, Lynn M. Resler ${ }^{2}$, Robert E. Keane ${ }^{3}$, Elizabeth R. Pansing ${ }^{1}$, \\ Andrew J. Andrade ${ }^{1}$ and Aaron C. Wagner ${ }^{1}$ \\ Received: 11 November 2015; Accepted: 6 January 2016; Published: 19 January 2016 \\ Academic Editor: Eric J. Jokela \\ 1 Department of Integrative Biology, CB 171, University of Colorado Denver, P.O. Box 173364, Denver, \\ CO 80217, USA; elizabeth.pansing@ucdenver.edu (E.R.P.); andrew.andrade@ucdenver.edu (A.J.A.); \\ aaron.wagner@ucdenver.edu (A.C.W.) \\ 2 Department of Geography, Virginia Tech, 115 Major Williams Hall, Blacksburg, VA 24061, USA; \\ resler@vt.edu \\ 3 U.S. Forest Service, Rocky Mountain Research Station, Missoula Fire Sciences Laboratory, \\ 5775 US Hwy 10 West, Missoula, MT 59808, USA; rkeane@fs.fed.us \\ * Correspondence: diana.tomback@ucdenver.edu; Tel.: +1-303-556-2657; Fax: +1-303-556-4352
}

\begin{abstract}
Whitebark pine (Pinus albicaulis) has the largest and most northerly distribution of any white pine (Subgenus Strobus) in North America, encompassing $18^{\circ}$ latitude and $21^{\circ}$ longitude in western mountains. Within this broad range, however, whitebark pine occurs within a narrow elevational zone, including upper subalpine and treeline forests, and functions generally as an important keystone and foundation species. In the Rocky Mountains, whitebark pine facilitates the development of krummholz conifer communities in the alpine-treeline ecotone (ATE), and thus potentially provides capacity for critical ecosystem services such as snow retention and soil stabilization. The invasive, exotic pathogen Cronartium ribicola, which causes white pine blister rust, now occurs nearly rangewide in whitebark pine communities, to their northern limits. Here, we synthesize data from 10 studies to document geographic variation in structure, conifer species, and understory plants in whitebark pine treeline communities, and examine the potential role of these communities in snow retention and regulating downstream flows. Whitebark pine mortality is predicted to alter treeline community composition, structure, and function. Whitebark pine losses in the ATE may also alter response to climate warming. Efforts to restore whitebark pine have thus far been limited to subalpine communities, particularly through planting seedlings with potential blister rust resistance. We discuss whether restoration strategies might be appropriate for treeline communities.
\end{abstract}

Keywords: Pinus albicaulis; alpine-treeline ecotone; biodiversity; community structure; keystone species; foundation species; ecosystem services; exotic pathogen; white pine blister rust; restoration

\section{Introduction}

Temperate zone coniferous forests are important contributors to global annual net primary productivity, standing biomass, and biodiversity ([1] and references therein). In North America, biodiversity assessments of temperate coniferous forest ecoregions range from regionally outstanding to globally outstanding [2]. Two extensive and important temperate coniferous forest ecoregions are the North Central Rockies Forests and South Central Rockies Forests, which include some of the world's most pristine wildlands, such as the Canadian Rockies, Northern Continental Divide Ecosystem, 
and the Greater Yellowstone Ecosystem. Although conifer diversity itself is low to moderate within these ecoregions, the composition and structure of each forest community type vary across steep moisture, topographic, and elevational gradients. Furthermore, wildfire is a recurring disturbance in Rocky Mountain forests, varying in intensity and return intervals across landscapes and leading to considerable community heterogeneity [3-6]. Variation among and within community types, as well as unique landscape community associations, are additive in contribution to regional biodiversity [7].

Community level biodiversity in these ecoregions, however, is not secure. These areas are epicenters for many of the forest health problems confronting western North American forests, including warming temperatures leading to reduced snowpack levels, earlier snowmelt [8], increased wildfire frequency [9], elevated tree mortality [10], as well as outbreaks of forest insects and pathogens [11,12], and ecosystem impacts of invasive pests, pathogens and plants [13]. These combined effects are predicted to push some forests over thresholds of resilience into new vegetation types, with a loss of forest ecosystem services [14].

Whitebark pine (Pinus albicaulis Engelm.) communities, which contribute significantly to western forest biodiversity, reach their distributional center of abundance in the North Central Rockies and South Central Rockies ecoregions [15]. They are impacted by a number of these forest health challenges across much of their range, but especially in the U.S. northwest and in the adjacent southwestern region of Canada [15,16]. In 2011, the species was designated a candidate under the U. S. Endangered Species Act and in 2012 declared endangered in Canada under the Species at Risk Act $[17,18]$. The two major factors cited in the species' decline were widespread mortality from white pine blister rust, a non-native disease caused by the fungal pathogen Cronartium ribicola J.C Fisch. in Rabh., which has spread nearly rangewide, and unprecedented outbreaks of mountain pine beetle (Dendroctonus ponderosae Hopkins). Other factors included altered fire regimes and advancing succession in seral communities from fire suppression efforts, and climate change [15,17,18].

Whitebark pine depends on the bird, Clark's nutcracker (Nucifraga columbiana (Wilson), for seed dispersal, which influences the pine's ecology, distribution, and population biology [19-21]. In late summer and fall, nutcrackers store ripe pine seeds throughout the montane landscape for future retrieval. Unretrieved seeds may germinate following snowmelt or rain, leading to regeneration (e.g., 22). Seed dispersal by nutcrackers to recently burned, disturbed, remote, or high elevation terrain, coupled with seedling tolerance of extreme conditions and harsh seedbeds, leads to whitebark pine regeneration early in succession and to whitebark pine establishment at the highest forest limits [20-22]. This coevolved, mutualistic seed dispersal system is related to the stone pine complex of Eurasia, which is dependent on seed dispersal by the Eurasian and large-spotted nutcrackers (Nucifraga caryocates L. and N. multipunctata Gould) [19].

Encompassing about $18^{\circ}$ latitude and $21^{\circ}$ longitude, whitebark pine has the largest latitudinal distribution of any North American five-needle white pine (Subgenus Strobus, Section Quinquefoliae, subsection Strobus [23]) but the narrowest elevational distribution comprising upper subalpine zone to treeline (Figure 1) [24]. The restricted elevational range results partly because whitebark pine is a "stress-tolerator" characterized by generally slow-growth, moderate shade-intolerance, and poor competitive abilities $[24,25]$. Whitebark pine community composition and dynamics change in relation to site characteristics: On comparatively warm, moist productive sites, communities are successional, with whitebark pine a minor component [26]. Although whitebark pine may persist into late seral stages in these communities, its overall basal area declines with time ([15] and references therein). These seral communities are disturbed periodically by fire. As sites become increasingly cold, and dry, with poorer soils, whitebark pine increases in community representation, becomes a self-replacing co-climax species, and then dominates as a self-replacing climax (late successional) species. Seral whitebark pine communities are most prevalent within the Rocky Mountain distribution, whereas the primary community types in the Pacific distribution are climax and treeline. 


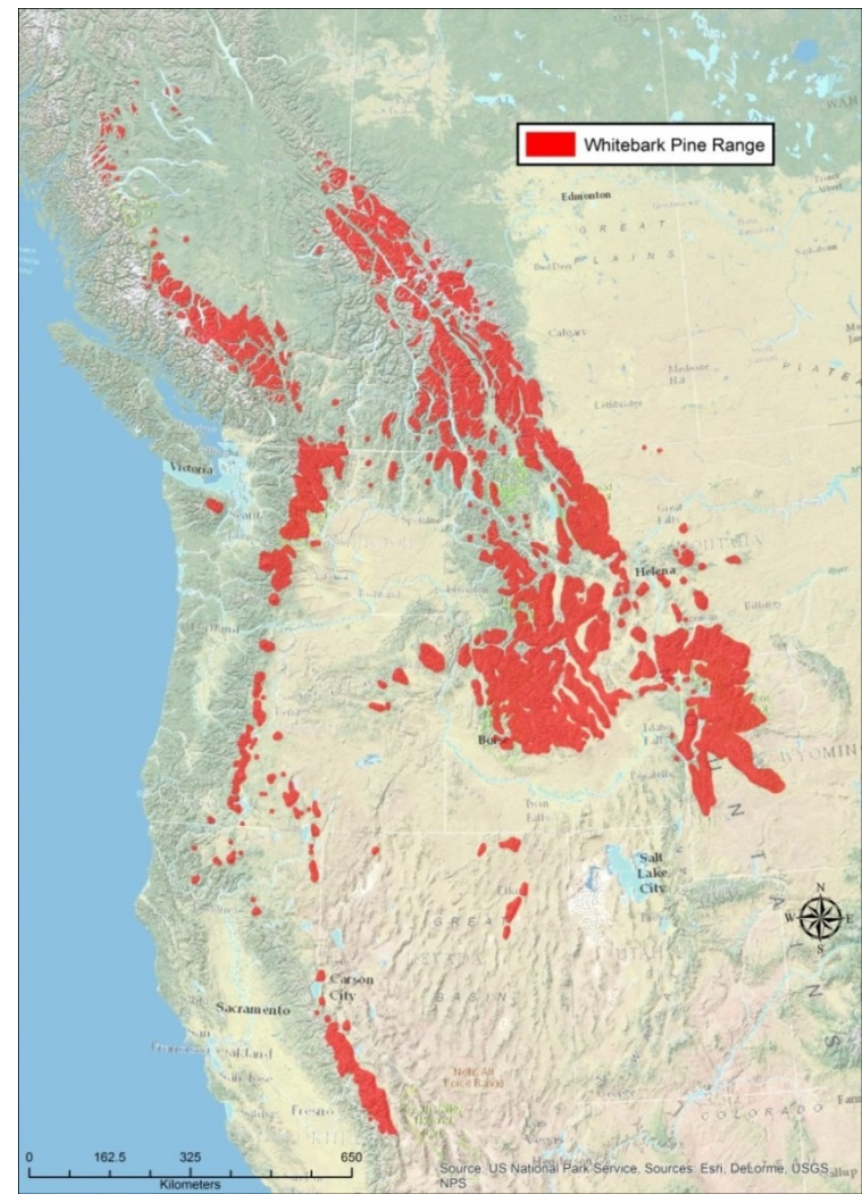

Figure 1. The distribution of whitebark pine (Pinus albicaulis Engelm.). (Source: Whitebark Pine Ecosystem Foundation [27]).

Across its range, whitebark pine functions as a foundation and keystone species [15,28]. A foundation species is regarded as "... disproportionately important to the continued maintenance of the existent community structure" ([29] p. 86). Loss of a foundational species profoundly alters structure, composition, and function [30]. Keystone species promote community diversity disproportionately in relation to their abundance through interactions with other species [31,32]. Foundation and keystone functions previously identified for whitebark pine are described in Table 1. Many of these functions result from highly effective seed dispersal by nutcrackers in combination with exceptionally hardy seedlings that rapidly develop deep tap roots and tolerate exposed, droughty sites $[22,24,28]$. Whitebark pine communities also provide ecosystem services, defined as ecosystem functions that benefit humans (e.g., [33]). Ecosystem services are divided into four general categories: provisioning services (e.g., food, timber), cultural services (e.g., recreation, inspiration), regulating services (e.g., preventing floods or disease), support services (e.g., clean water, pollination) [34]. Ecosystem services specific to whitebark pine are listed in Table 1.

Snowpack retention and snowmelt protraction are ecosystem services associated with whitebark pine subalpine forest and treeline communities (Table 1) [28,35]. Whitebark pine often grows in soils that hold little water and support sparse vegetation at elevations with high precipitation and snow accumulation, leading to copious run-off [35]. Because snow is the dominant form of precipitation in Rocky Mountain ecosystems, hydrologic processes are largely governed by snowpack dynamics, including snow deposition, redistribution, and runoff [41-44]. It has been inferred that high elevation whitebark pine communities create conditions that delay snowmelt and therefore downstream flow until late in the season, when it is most valuable for down-valley irrigation needs $[28,35]$. 
However, specific connections between whitebark pine communities and hydrologic processes lack empirical support.

Table 1. Keystone interactions (K), foundational functions (F), and ecosystem services provided by whitebark pine [15,16,28,35-38]. Ecosystem services to Native Americans [39,40].

\section{Keystone Interactions and Foundational Functions}

- Wide latitudinal distribution and environmental tolerances with a gradient of community types lead to forest, understory plant, and animal community diversity at local, regional, and range-wide scales. K, F

- Community structure provides wildlife shelter, perching and nesting sites, burrows, and food at high elevations. $\mathbf{K}$

- Large, nutritious seeds provide an important wildlife food source for songbirds, upland game birds, squirrels and mice, bears, and foxes. $\mathbf{K}$

- Hardiness of seedlings coupled with seed dispersal by Clark's nutcrackers leads to early whitebark pine regeneration after fire and other disturbance across slope aspects and elevations, which in turn mitigates harsh conditions and leads to community development through facilitation interactions. F

- Hardiness of seedlings leads to tolerance of harsh upper subalpine sites and establishment of whitebark pine. A less hardy tree may then establish in its lee, through facilitation, which fosters biodiversity. K, F

- Tolerance of harsh, windy sites, coupled with seed dispersal by Clark's nutcrackers to treeline microsites, leads to solitary whitebark pine facilitating leeward tree establishment, which enables tree island community development. F

Ecosystem services

- As the principal conifer growing at the highest elevations in the upper subalpine and at treeline, whitebark pine stands shade snowpack and slow snowmelt, which leads to protracted runoff and longer seasonal downstream flow. This potentially benefits agriculture and ranching.

- $\quad$ By growing at high elevations on harsh sites with rocky substrates, whitebark pine tree roots take up water, regulating flows, stabilize soils, and reduce soil erosion, potentially resulting in higher quality water with less sediment in downstream flows.

- Whitebark pine communities are associated with high elevation recreational activities in national forests, wilderness areas, national parks, and ski areas. On harsh sites, individual trees assume massive, twisted wind-sculpted forms, which to visitors symbolize survival against the elements and contribute uplifting aesthetics to the high-mountain experience.

- The large seeds of whitebark pine were an important traditional food for several Rocky Mountain Native American tribes. Tribes also used the inner bark of whitebark pine as a food source in spring or early summer.

In the North Central Rockies and South Central Rockies ecoregions, whitebark pine is a dominant krummholz species in many alpine-treeline ecotone (ATE) communities, especially on the harsh eastern front of the Rocky Mountains [38,45]. Within these communities, whitebark pine is not only the most abundant solitary species in these regions but also the species most often at the exposed windward edge of tree islands and thus the most common species to initiate tree island formation, a foundational function (Table 1). Whitebark pine is hardier under conditions of poor soils, wind, low snow cover, and drought than associated treeline conifers (e.g., [46]), and its leeward microsites provide a protective microclimate and reduced sky exposure, favoring the establishment of other trees [47].

The foundation and keystone functions of whitebark pine are generally recognized [15-18,28], as well is the range of forest biodiversity represented by whitebark pine subalpine communities [24,26,37]. We ask whether there may also be geographic variation in treeline community composition and structure, thus providing a more complete understanding of the range of forest types and community biodiversity represented by whitebark pine. Secondly, we review hydrologic processes at high elevations to determine whether ATE whitebark pine communities potentially retain snowpack and regulate local streamflows, which would provide inferential support for this ecosystem service in the absence of empirical evidence. Specifically, we examine both (1) geographic variation in the structure and composition of whitebark pine krummholz ATE communities and (2) geographic variation in dominant co-occurring "understory" plants, using information from our collective recent studies in the North Central and South Central Rockies. We also (3) review the literature for information on the contribution of treeline communities to snow retention and local hydrological processes, and connect 
this information to ATE whitebark pine communities. Furthermore, previous studies in the ATE across the distribution of whitebark pine indicate that $C$. ribicola is present in nearly every community examined [45], with the highest infection levels in the ATE of Glacier National Park [38,48,49]. Blister rust potentially threatens both the biodiversity supported by whitebark pine as a keystone and foundation species and the ecosystem services that whitebark pine communities provide. Finally, we explore the issue of whether restoration efforts are feasible or desirable for treeline communities.

\section{Study Areas and Methods}

Whitebark pine in the Rocky Mountains ranges from about $42^{\circ}$ latitude in Wyoming, USA, to $54^{\circ}$ latitude in British Columbia and Alberta, Canada. Summary data presented on the structure and composition of krummholz whitebark pine ATE communities come from ten studies either previously published or in preparation, spanning the latitudinal range of whitebark pine in the Rocky Mountains (Table 2, Figure 2).

Community assessment methods varied by study in plot size $\left(225 \mathrm{~m}^{2} v s .500 \mathrm{~m}^{2}\right)$, number of plots (2-30), total area sampled $\left(750 \mathrm{~m}^{2}\right.$ to $6750 \mathrm{~m}^{2}$; median $\left.=4500 \mathrm{~m}^{2}\right)$, and whether the plots were randomly or non-randomly distributed. Seven studies were based on a randomized distribution of study plots. For non-random sampling, plots were distributed within a defined aspect and slope at the highest elevations of the ATE that could be safely accessed; whereas, for random sampling, random points for plot initiation were selected a priori and stratified by aspect based on GIS or R [50]. We sampled non-randomly in the Willmore Wilderness Park, Stanley Glacier, and Gibbon Pass study areas, where surveys had to be completed at a specific location within a limited timeframe.

Sampled conifers were predominantly of krummholz growth form, or a mixture of krummholz with upright, flagged stems, and an occasional erect tree, especially alpine larch (Larix lyallii Parl.). Trees occurred either solitarily or in tree islands, where krummholz tree canopies were contiguous, and sometimes mixed with flagged branches and more upright forms. In all study areas, the following data were collected per plot: tree species richness S (not including shrub forms, such as Juniperus spp.), number of solitary trees per species, number of tree islands, species composition of each tree island by species presence (not by total number of each species), length of each tree island (to a maximum of $35 \mathrm{~m}$ ), and species of the most windward tree (likely tree island initiator) for each tree island.

We calculated descriptive statistics for each study area using either Microsoft Excel or R [50] for plot level variables for numbers of solitary trees and numbers of tree islands per $\mathrm{m}^{2}$, which we scaled up to densities per hectare, and tree island lengths. We report species richness among all solitary trees and tree islands and relative proportions of tree island initiators by species. The Shannon Index was used to calculate the community diversity of solitary trees for each study area:

$$
H=-\sum_{i=1}^{S}\left(p_{i}\right)\left(\log _{2} p_{i}\right)
$$

Information on understory plant dominants comes from our collaborative studies in these study areas. We reviewed the literature for information on the role of treeline conifer communities in snow retention, and thus provision of this important ecosystem service.

We tested data for normality. We performed linear regression to determine if the proportion of solitary trees represented by whitebark pine predicted the proportion of tree islands with whitebark pine as the initiator, and we performed Spearman rank correlation analysis to determine if tree island density correlated with tree island length [50]. 
Table 2. Study areas across the latitudinal range of whitebark pine in the Rocky Mountains, and sampling methods providing information on structure and composition of treeline communities.

\begin{tabular}{|c|c|c|c|c|c|c|}
\hline Study Area & $\begin{array}{l}\text { Latitude and } \\
\text { Longitude }\end{array}$ & Elevation $\mathrm{m}$ & Aspect & Plot Size, $N$ & $\begin{array}{l}\text { Area Sampled; } \\
\text { Distribution }\end{array}$ & Reference \\
\hline Willmore Wilderness Park; Alberta, Canada & $\begin{array}{c}53^{\circ} 46^{\prime} 0^{\prime \prime} \mathrm{N} \\
119^{\circ} 44^{\prime} 22^{\prime \prime} \mathrm{W}\end{array}$ & $1964-2175$ & SW, SE, E, NE & $\begin{array}{l}500 \mathrm{~m}^{2} \\
N=11\end{array}$ & $\begin{array}{c}5500 \mathrm{~m}^{2} ; \\
\text { non-random }\end{array}$ & Tomback and Resler, in preparation [51] \\
\hline Parker Ridge Banff National Park; Alberta, Canada & $\begin{array}{l}52^{\circ} 10^{\prime} 44^{\prime \prime} \mathrm{N} ; \\
117^{\circ} 06^{\prime} 24^{\prime \prime} \mathrm{W}\end{array}$ & 2100 & NE, NW, S, SE, SW & $\begin{array}{l}225 \mathrm{~m}^{2} \\
N=20\end{array}$ & $\begin{array}{l}4500 \mathrm{~m}^{2} ; \\
\text { random }\end{array}$ & $\begin{array}{l}\text { Resler et al. [52]; Resler } \text { et al., } \\
\text { unpublished data [53] }\end{array}$ \\
\hline Gibbon Pass; Banff National Park, Alberta, Canada & $\begin{array}{l}51^{\circ} 11^{\prime} 15^{\prime \prime} \mathrm{N} ; \\
115^{\circ} 56^{\prime} 12^{\prime \prime} \mathrm{W}\end{array}$ & $2389-2430$ & $\mathrm{SE}, \mathrm{NE}$ & $\begin{array}{l}500 \mathrm{~m}^{2} \\
N=3\end{array}$ & $\begin{array}{c}1500 \mathrm{~m}^{2} \\
\text { non-random }\end{array}$ & Tomback et al. [45] \\
\hline Stanley Glacier; Banff National Park, Alberta, Canada & $\begin{array}{l}51^{\circ} 11^{\prime} 13^{\prime \prime} \mathrm{N} \\
116^{\circ} 02^{\prime} 51^{\prime \prime} \mathrm{W}\end{array}$ & 1969-1997 & SW, NW & $\begin{array}{c}500 \mathrm{~m}^{2} \\
N=1 \\
250 \mathrm{~m}^{2} \\
N=1\end{array}$ & $\begin{array}{c}750 \mathrm{~m}^{2} \\
\text { non-random }\end{array}$ & Tomback et al. [45] \\
\hline $\begin{array}{l}\text { Divide Mountain/White Calf Mountain; Glacier } \\
\text { National Park, Blackfeet Indian Reservation, MT }\end{array}$ & $\begin{array}{c}48^{\circ} 39^{\prime} 25^{\prime \prime} \mathrm{N} \\
113^{\circ} 23^{\prime} 45^{\prime \prime} \mathrm{W} ; \\
48^{\circ} 38^{\prime} 20^{\prime \prime} \mathrm{N} ; \\
113^{\circ} 24^{\prime} 08^{\prime \prime} \mathrm{W}\end{array}$ & $1920-2272$ & NE, NW, W, SW & $\begin{array}{l}225 \mathrm{~m}^{2} \\
N=30\end{array}$ & $\begin{array}{l}6750 \mathrm{~m}^{2} \\
\text { random }\end{array}$ & Smith-McKenna et al. [48] \\
\hline Line Creek; Custer National Forest, MT & $\begin{array}{l}45^{\circ} 01^{\prime} 47^{\prime \prime} \mathrm{N} ; \\
109^{\circ} 24^{\prime} 09^{\prime \prime} \mathrm{W} \\
\end{array}$ & 2950 & NE & $\begin{array}{l}225 \mathrm{~m}^{2} \\
N=30\end{array}$ & $\begin{array}{l}6750 \mathrm{~m}^{2} \\
\text { random }\end{array}$ & Smith-McKenna et al. [48] \\
\hline Tibbs Butte; Shoshone National Forest, WY & $\begin{array}{c}44^{\circ} 56^{\prime} 28^{\prime \prime} \mathrm{N} \\
109^{\circ} 26^{\prime} 39.69^{\prime \prime} \mathrm{W}\end{array}$ & $2983-3238$ & $\mathrm{E}, \mathrm{NE}$ & $\begin{array}{l}225 \mathrm{~m}^{2} \\
N=12\end{array}$ & $\begin{array}{l}2700 \mathrm{~m}^{2} \\
\text { random }\end{array}$ & Wagner et al., in preparation [54] \\
\hline $\begin{array}{l}\text { Paintbrush Divide/Holly Lake; } \\
\text { Grand Teton National Park, WY }\end{array}$ & $\begin{array}{r}43^{\circ} 47^{\prime} 34^{\prime \prime} \mathrm{N} ; \\
110^{\circ} 47^{\prime} 54^{\prime \prime} \mathrm{W} \\
\end{array}$ & $3055-3289$ & NW, SW, NE & $\begin{array}{l}225 \mathrm{~m}^{2} \\
N=20\end{array}$ & $\begin{array}{l}4500 \mathrm{~m}^{2} ; \\
\text { random }\end{array}$ & Resler and Shao, unpublished data [55] \\
\hline $\begin{array}{l}\text { Hurricane Pass/Avalanche Basin; } \\
\text { Grand Teton National Park, WY }\end{array}$ & $\begin{array}{l}43^{\circ} 43^{\prime} 41^{\prime \prime} \mathrm{N} ; \\
110^{\circ} 51^{\prime} 02^{\prime \prime} \mathrm{W}\end{array}$ & $3045-3204$ & NW, SW, NE & $\begin{array}{l}225 \mathrm{~m}^{2} \\
N=20\end{array}$ & $\begin{array}{l}4500 \mathrm{~m}^{2} \\
\text { random }\end{array}$ & Resler and Shao, unpublished data [55] \\
\hline Christina Lake; Shoshone National Forest, WY & $\begin{array}{l}42^{\circ} 35^{\prime} 35^{\prime \prime} \mathrm{N} \\
108^{\circ} 58^{\prime} 24^{\prime \prime} \mathrm{W}\end{array}$ & $3200-3400$ & SW, SE, NE & $\begin{array}{l}225 \mathrm{~m}^{2} \\
N=30\end{array}$ & $\begin{array}{l}6,750 \mathrm{~m}^{2} \\
\text { random }\end{array}$ & Wagner et al., in preparation [54] \\
\hline
\end{tabular}




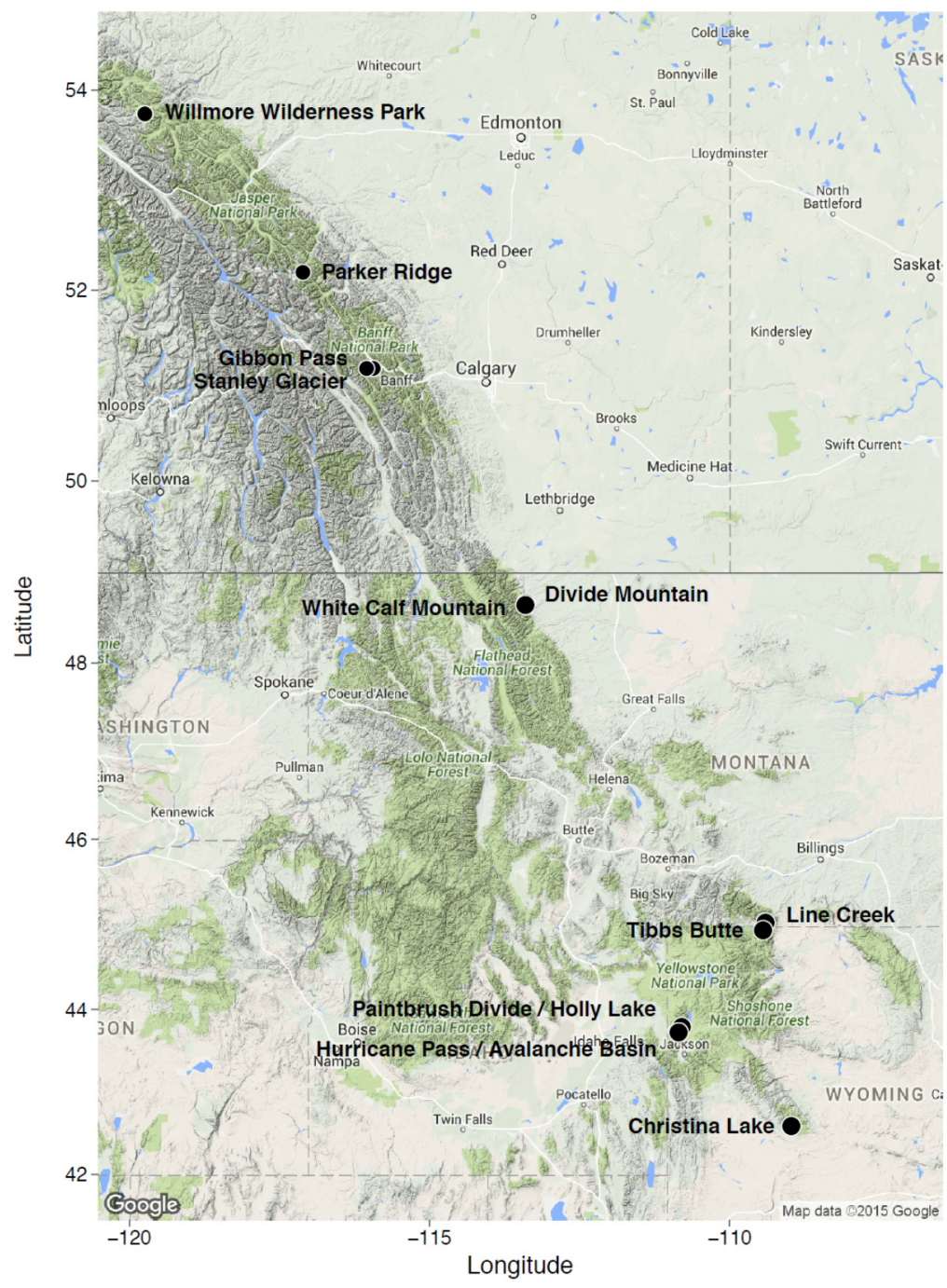

Figure 2. The locations of the ten alpine-treeline ecotone (ATE) community study areas encompassing the geographic range of whitebark pine in the Northern and Central Rocky Mountains. These areas provided the comparative data used in this study. See Table 2 for additional information about each study area.

\section{Results}

\subsection{Geographic Variation in ATE Community Composition and Structure}

The ATE communities in all study areas comprised both small, scattered solitary krummholz trees interspersed among tree islands and predominantly krummholz tree islands. Solitary trees occurred in open terrain among tree islands but also as non-contiguous satellites around tree islands. Median densities of solitary trees per hectare, with all species combined, ranged from 160.00 trees/ha at Gibbon Pass to 977.78 trees/ha at Divide Mountain (Table 3). With the exception of Gibbon Pass, the highest densities of solitary trees occurred within the northern study areas. The median densities of tree islands ranged from 0.00/ha at both Tibbs Butte and Christina Lake to 130.00/ha at Stanley Glacier, but half of the median values were $c a .80 /$ ha (Table 3). Again, the highest median densities were generally in the northern study areas. At Stanley Glacier, Divide Mountain, Paintbrush Divide/Holly Lake, and Hurricane Pass/Avalanche Basin, we encountered one or more tree islands longer than $35 \mathrm{~m}$ in length (Table 3). Median tree island lengths ranged from $2.59 \mathrm{~m}$ to $7.68 \mathrm{~m}$ among the study areas, with no obvious trends. 
Table 3. Tree species richness, conifer composition, solitary tree density and tree island density, tree island length, and understory dominants for the ten study areas listed in Table 2. Conifer abbreviations are as follows: PIAL = whitebark pine (Pinus albicaulis), ABLA = subalpine fir (Abies lasiocarpa), PIEN = Engelmann spruce (Picea engelmannii), PICO = lodgepole pine (Pinus contorta), LALY = alpine larch (Larix lyallii), and PSME = Douglas-fir (Pseudotsuga menziesii).

\begin{tabular}{|c|c|c|c|c|c|c|c|}
\hline Location & $\begin{array}{l}\text { Tree Spec } \\
\text { Solitary }\end{array}$ & $\begin{array}{r}\text { es Richness S } \\
\text { Tree Islands }\end{array}$ & $\begin{array}{l}\text { Median, Min, Max, } \\
\text { No. Solitary Trees/ha, } \\
\text { total Solitary Trees } n\end{array}$ & $\begin{array}{c}\text { Shannon } \\
\text { Diversity Index } \\
\text { for Solitary Trees }\end{array}$ & $\begin{array}{l}\text { Median, Min, Max, } \\
\text { Tree Islands/ha, } \\
\text { Total Tree Islands } n\end{array}$ & $\begin{array}{c}\text { Median, Min, } \\
\text { Max Length m, } \\
\text { Total Tree Islands } n\end{array}$ & Dominant Understory Species \\
\hline $\begin{array}{c}\text { Willmore } \\
\text { Wilderness Park }\end{array}$ & $\begin{array}{c}4 \\
\text { PIAL } \\
\text { ABLA } \\
\text { PIEN } \\
\text { PICO }\end{array}$ & $\begin{array}{c}4 \\
\text { PIAL } \\
\text { ABLA } \\
\text { PIEN } \\
\text { PICO }\end{array}$ & $\begin{array}{c}780.00 \\
140.00 \\
2080.00 \\
n=485\end{array}$ & 0.724 & $\begin{array}{c}80.00 \\
20.00 \\
200.00 \\
n=49\end{array}$ & $\begin{array}{c}2.59 \\
0.10 \\
9.60 \\
n=48\end{array}$ & $\begin{array}{c}\text { Dryas integrifolia Vahl } \\
\text { Cassiope mertensiana (Bong.) G. Don } \\
\text { Empetrum nigrum L. } \\
\text { Phyllodoce glanduliflora (Hook.) Coville } \\
\text { Rhododendron albiflorum Hook. } \\
\text { Menziesia ferruginea Sm. } \\
\text { Vaccinium membranaceum Douglas ex Torr. }\end{array}$ \\
\hline Parker Ridge & $\begin{array}{c}3 \\
\text { PIAL } \\
\text { ABLA } \\
\text { PIEN }\end{array}$ & $\begin{array}{c}3 \\
\text { PIAL } \\
\text { ABLA } \\
\text { PIEN }\end{array}$ & $\begin{array}{c}777.78 \\
88.89 \\
2400.00 \\
n=321\end{array}$ & 0.830 & $\begin{array}{c}88.89 \\
0 \\
222.22 \\
n=49\end{array}$ & $\begin{array}{c}2.70 \\
0.04 \\
19.90 \\
n=49\end{array}$ & $\begin{array}{l}\text { Dryas octopetala L. } \\
\text { Silene acaulis (L.) Jacq. }\end{array}$ \\
\hline Gibbon Pass & $\begin{array}{c}4 \\
\text { PIAL } \\
\text { ABLA } \\
\text { PIEN } \\
\text { LALY }\end{array}$ & $\begin{array}{c}4 \\
\text { PIAL } \\
\text { ABLA } \\
\text { PIEN } \\
\text { LALY }\end{array}$ & $\begin{array}{l}160.00 \\
120.00 \\
1760.00 \\
n=103\end{array}$ & 1.312 & $\begin{array}{c}80.00 \\
80.00 \\
100.00 \\
n=12\end{array}$ & $\begin{array}{c}4.35 \\
0.65 \\
11.60 \\
n=12\end{array}$ & $\begin{array}{l}\text { Dryas octopetala L. } \\
\text { Silene acaulis (L.) Jacq. }\end{array}$ \\
\hline Stanley Glacier & $\begin{array}{c}3 \\
\text { PIAL } \\
\text { ABLA } \\
\text { PIEN }\end{array}$ & $\begin{array}{c}3 \\
\text { PIAL } \\
\text { ABLA } \\
\text { PIEN }\end{array}$ & $\begin{array}{c}720.00 \\
240.00 \\
1200.00 \\
n=32\end{array}$ & 0.769 & $\begin{array}{c}130.00 \\
60.00 \\
200.00 \\
n=8\end{array}$ & $\begin{array}{c}7.34^{*} \\
1.00 \\
>35.00 \\
n=8\end{array}$ & $\begin{array}{l}\text { Dryas octopetala L. } \\
\text { Silene acaulis (L.) Jacq. }\end{array}$ \\
\hline Divide Mountain & $\begin{array}{c}4 \\
\text { PIAL } \\
\text { ABLA }\end{array}$ & $\begin{array}{c}4 \\
\text { PIAL } \\
\text { ABLA }\end{array}$ & $\begin{array}{c}977.78 \\
88.89\end{array}$ & 0.778 & $\begin{array}{c}111.00 \\
0.00\end{array}$ & $\begin{array}{l}5.40 \\
0.40\end{array}$ & $\begin{array}{c}\text { Dryas octopetala L. } \\
\text { Arctostaphylos uva-ursi (L.) Spreng. } \\
\text { Hedysarum sulphurescens Rydb. } \\
\text { Oxytropis sericea Nutt. } \\
\text { Silene acaulis (L.) Jacq. }\end{array}$ \\
\hline $\begin{array}{l}\text { White Calf } \\
\text { Mountain }\end{array}$ & $\begin{array}{l}\text { PIEN } \\
\text { PSME }\end{array}$ & $\begin{array}{l}\text { PIEN } \\
\text { PSME }\end{array}$ & $\begin{array}{l}3911.11 \\
n=367\end{array}$ & & $\begin{array}{l}355.55 \\
n=84\end{array}$ & $\begin{array}{l}>35.00 \\
n=84\end{array}$ & $\begin{array}{l}\text { Arctostaphylos uva-ursi (L.) Spreng. } \\
\text { Achillea millefolium L. } \\
\text { Hedysarum sulphurescens Rydb. } \\
\text { Potentilla diversifolia Lehm. }\end{array}$ \\
\hline
\end{tabular}


Table 3. Cont

\begin{tabular}{|c|c|c|c|c|c|c|c|}
\hline Location & $\begin{array}{l}\text { Tree Spec } \\
\text { Solitary }\end{array}$ & $\begin{array}{l}\text { Tree Islands } \\
\text { Tress S }\end{array}$ & $\begin{array}{l}\text { Median, Min, Max, } \\
\text { No. Solitary Trees/ha, } \\
\text { total Solitary Trees } n\end{array}$ & $\begin{array}{c}\text { Shannon } \\
\text { Diversity Index } \\
\text { for Solitary Trees }\end{array}$ & $\begin{array}{l}\text { Median, Min, Max, } \\
\text { Tree Islands/ha, } \\
\text { Total Tree Islands } n\end{array}$ & $\begin{array}{c}\text { Median, Min, } \\
\text { Max Length m, } \\
\text { Total Tree Islands } n\end{array}$ & Dominant Understory Species \\
\hline Line Creek & $\begin{array}{c}5 \\
\text { PIAL } \\
\text { ABLA } \\
\text { PIEN } \\
\text { PSME } \\
\text { PICO }\end{array}$ & $\begin{array}{l}5 \\
\text { PIAL } \\
\text { ABLA } \\
\text { PIEN } \\
\text { PSME } \\
\text { PICO }\end{array}$ & $\begin{array}{c}533.33 \\
88.89 \\
1733.33 \\
n=244\end{array}$ & 0.695 & $\begin{array}{c}88.89 \\
0444.44 \\
n=62\end{array}$ & $\begin{array}{c}4.50 \\
0.76 \\
16.88 \\
n=62\end{array}$ & $\begin{array}{l}\text { Agoseris glauca (Pursh) Raf. } \\
\text { Aster alpigenus (Torr. \& A. Gray) A. Gray } \\
\text { Geum rossii (R. Br.) Ser. } \\
\text { Potentilla diversifolia Lehm. } \\
\text { Lupinus argenteus Pursh }\end{array}$ \\
\hline Tibbs Butte & $\begin{array}{c}2 \\
\text { PIAL } \\
\text { PIEN }\end{array}$ & $\begin{array}{c}3 \\
\text { PIAL } \\
\text { ABLA } \\
\text { PIEN }\end{array}$ & $\begin{array}{c}222.22 \\
44.44 \\
1422.22 \\
n=88\end{array}$ & 0.249 & $\begin{array}{c}0.00 \\
0.00 \\
88.89 \\
n=3\end{array}$ & $\begin{array}{c}7.68 \\
0.76 \\
14.07 \\
n=3\end{array}$ & $\begin{array}{l}\text { Geum rossii (R. Br.) Ser. } \\
\text { Potentilla diversifolia Lehm. } \\
\text { Saxifrage spp. } \\
\text { Graminoids }\end{array}$ \\
\hline $\begin{array}{l}\text { Paintbrush } \\
\text { Divide/Holly } \\
\text { Lake }\end{array}$ & $\begin{array}{c}3 \\
\text { PIAL } \\
\text { ABLA } \\
\text { PIEN }\end{array}$ & $\begin{array}{c}3 \\
\text { PIAL } \\
\text { ABLA } \\
\text { PIEN }\end{array}$ & $\begin{array}{c}550.71 \\
0.00 \\
1497.93 \\
n=282\end{array}$ & 0.828 & $\begin{array}{c}88.11 \\
0.00 \\
308.40 \\
n=45\end{array}$ & $\begin{array}{c}3.35 \\
0.11 \\
> \\
35.00 \\
n=45\end{array}$ & $\begin{array}{c}\text { Arctostaphylos uva-ursi (L.) } \\
\text { Spreng. Myosotis asiatica (Vesterg.) } \\
\text { Schischkin \& Sergievskaja } \\
\text { Senecio spp. } \\
\text { Silene acaulis (L.) Jacq. }\end{array}$ \\
\hline $\begin{array}{c}\text { Hurricane } \\
\text { Pass/Avalanche } \\
\text { Basin }\end{array}$ & $\begin{array}{c}3 \\
\text { PIAL } \\
\text { ABLA } \\
\text { PIEN }\end{array}$ & $\begin{array}{l}4 \\
\text { PIAL } \\
\text { ABLA } \\
\text { PIEN } \\
\text { PSME }\end{array}$ & $\begin{array}{c}242.31 \\
0.00 \\
793.02 \\
n=138\end{array}$ & 1.069 & $\begin{array}{c}66.08 \\
0.00 \\
352.45 \\
n=45\end{array}$ & $\begin{aligned} & 7.30 \\
& 0.98 \\
&> 35.00 \\
& n=45 \\
&\end{aligned}$ & $\begin{array}{l}\text { Dryas octopetala L. } \\
\text { Senecio spp. } \\
\text { Silene acaulis (L.) Jacq. } \\
\text { Graminoids }\end{array}$ \\
\hline Christina Lake & $\begin{array}{c}3 \\
\text { PIAL } \\
\text { ABLA } \\
\text { PIEN }\end{array}$ & $\begin{array}{c}3 \\
\text { PIAL } \\
\text { ABLA } \\
\text { PIEN }\end{array}$ & $\begin{array}{c}244.44 \\
0.00 \\
2000.00 \\
n=247\end{array}$ & 0.421 & $\begin{array}{c}0.00 \\
0.00 \\
444.44 \\
n=41\end{array}$ & $\begin{array}{l}3.39 \\
1.14 \\
29.07 \\
n=41\end{array}$ & $\begin{array}{c}\text { Geum rossii (R. Br.) Ser. } \\
\text { Pteryxia hendersonii (J.M. Coult. \& Rose) } \\
\text { Mathias \& Constance } \\
\text { Phlox pulvinata (Wherry) Cronquist } \\
\text { Silene acaulis (L.) Jacq. }\end{array}$ \\
\hline
\end{tabular}


Among study areas, conifer species richness $S$ for solitary trees ranged from 2 to 5 and for tree islands from 3 to 5 , with the highest richness in the Line Creek study area (Table 3). With the exception of the Gibbon Pass and Tibbs Butte study areas, solitary trees and tree islands were composed primarily of whitebark pine (PIAL), subalpine fir (Abies lasiocarpa (Hook.) Nutt., ABLA), and Engelmann spruce (Picea engelmannii Parry ex Engelmann, PIEN). Small numbers of other species occurred in several study areas: lodgepole pine (Pinus contorta Douglas ex Loudon, PICO) in Willmore Wilderness Park and Line Creek, alpine larch (Larix lyallii Parl., LALY) in Gibbon Pass, and Douglas-fir (Pseudotsuga menziesii (Mirb.) Franco, PSME) in Divide Mountain and Line Creek (Table 3).

Among solitary trees, whitebark pine was the majority species in nine of the ten study areas, ranging from $93.2 \%$ at Tibbs Butte to $23.3 \%$ at Gibbon Pass-the latter an outlier in community composition (Figure 3). Species comprising the other solitary trees varied in relative abundance (Figure 3). At Gibbon Pass, Engelmann spruce dominated solitary trees, accounting for $37.9 \%$. In other study areas, Engelmann spruce ranged in percent occurrence among solitary trees from $5.3 \%$ at Christina Lake to $34.4 \%$ at Stanley Glacier. Subalpine fir ranged from 5.7\% at Christina Lake to $23.2 \%$ at Hurricane Pass / Avalanche Basin. At Gibbon Pass, alpine larch was the second most prevalent solitary tree after spruce, and at Line Creek, lodgepole pine was more common than subalpine fir. The Gibbon Pass and Hurricane Pass/Avalanche Basin study areas had the highest Shannon diversity indices for the solitary tree component of the ATE community, primarily because of greater evenness in relative occurrence among conifers (Table 3). The Tibbs Butte study area had the lowest Shannon diversity index, because of dominance by whitebark pine and a low species richness of 2 . One or more whitebark pine trees occurred across the highest proportion of tree islands than any other conifer species in all study areas except Parker Ridge and Gibbon Pass (Figure 4).

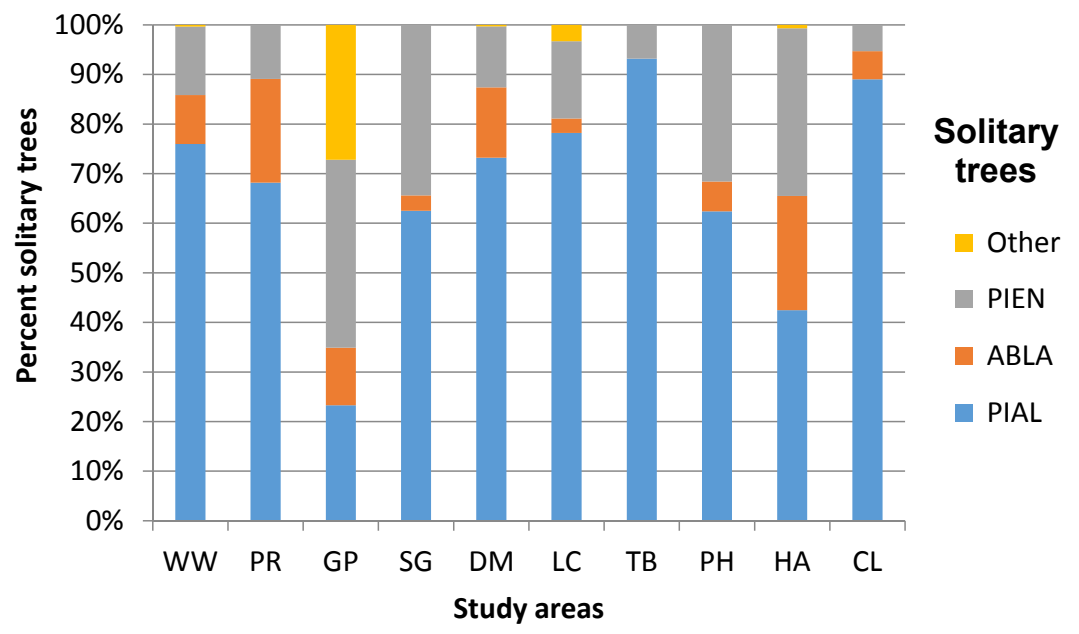

Figure 3. Percent composition of the solitary conifer tree community for each study area. See Table 3 for conifer species listed under "Other" for each study area. Conifer abbreviations are as follows: PIAL = whitebark pine (Pinus albicaulis), ABLA = subalpine fir (Abies lasiocarpa), PIEN = Engelmann spruce (Picea engelmannii), PICO = lodgepole pine (Pinus contorta), LALY = alpine larch (Larix lyallii), and PSME = Douglas-fir (Pseudotsuga menziesii). Study areas are abbreviation as follows: $\mathrm{WW}=$ Willmore Wilderness, $\mathrm{PR}=$ Parker Ridge, GP $=$ Gibbon Pass, SG $=$ Stanley Glacier, $\mathrm{DM}=$ Divide Mountain/White Calf Mountain, $\mathrm{LC}=$ Line Creek, TB = Tibbs Butte, $\mathrm{PH}=$ Paintbrush Divide/Holly Lake, HA = Hurricane Pass/Avalanche Basin, and CL = Christina Lake.

The relative proportions of tree islands with subalpine fir and Engelmann spruce varied across study areas from Line Creek north, but were comparable in study areas from Tibbs Butte south. Median tree island length varied from $2.59 \mathrm{~m}$ in the Willmore Wilderness study area to $7.68 \mathrm{~m}$ at Tibbs Butte; no latitudinal gradient was apparent, and median density of tree islands did not predict length of tree islands ( $\rho=0.191$ (95\% CI: $-0.572,0.881), N=10, p=0.598)$. 


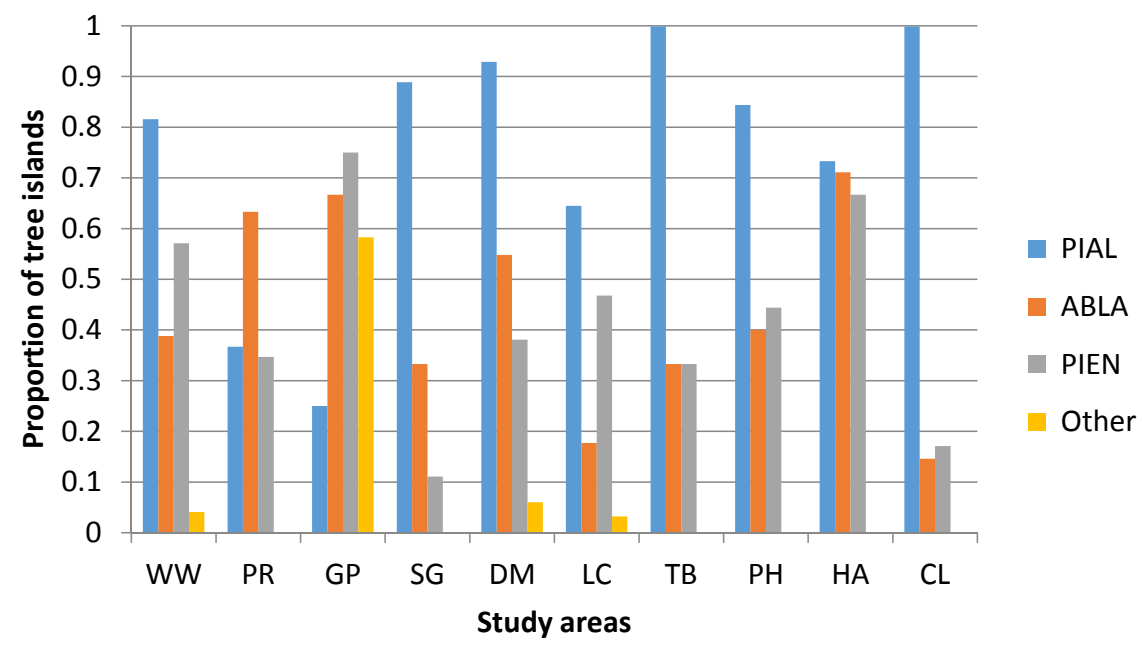

Figure 4. The proportion of tree islands within a study area with one or more individuals of each species. See Table 3 for conifer species listed under "Other", and Figure 3 for conifer species abbreviations and study area abbreviations.

Whitebark pine was the most frequently occurring windward conifer in tree islands, and thus presumably the majority tree island initiator, within five study areas: Stanley Glacier, Divide Mountain, Line Creek, Tibbs Butte, and Christina Lake (Figure 5). We examined whether the relative proportion of whitebark pine as a solitary tree predicted its relative proportion as a tree island initiator across study areas. We found that proportional abundance of whitebark pine among solitary trees predicted its proportional abundance as a tree island initiator $\left(F=8.724, r=0.722, R^{2}=0.522, d f=8, p=0.018\right)$. Several study areas, such as Willmore Wilderness Park, Parker Ridge, and Paintbrush Divide/Holly Lake, had high proportions of whitebark pine among solitary trees but not among tree island initiators (Figure 3). We tested the same data sets for subalpine fir and Engelmann spruce and found that proportional representation of these conifers as solitary trees did not as strongly predict their proportional abundance as tree island initiators $\left(F=5.192, r=0.627, R^{2}=0.3936, d f=8, p=0.0522\right.$, and $F=2.645, r=0.498$, $R^{2}=0.2485, d f=8, p=0.143$, respectively).

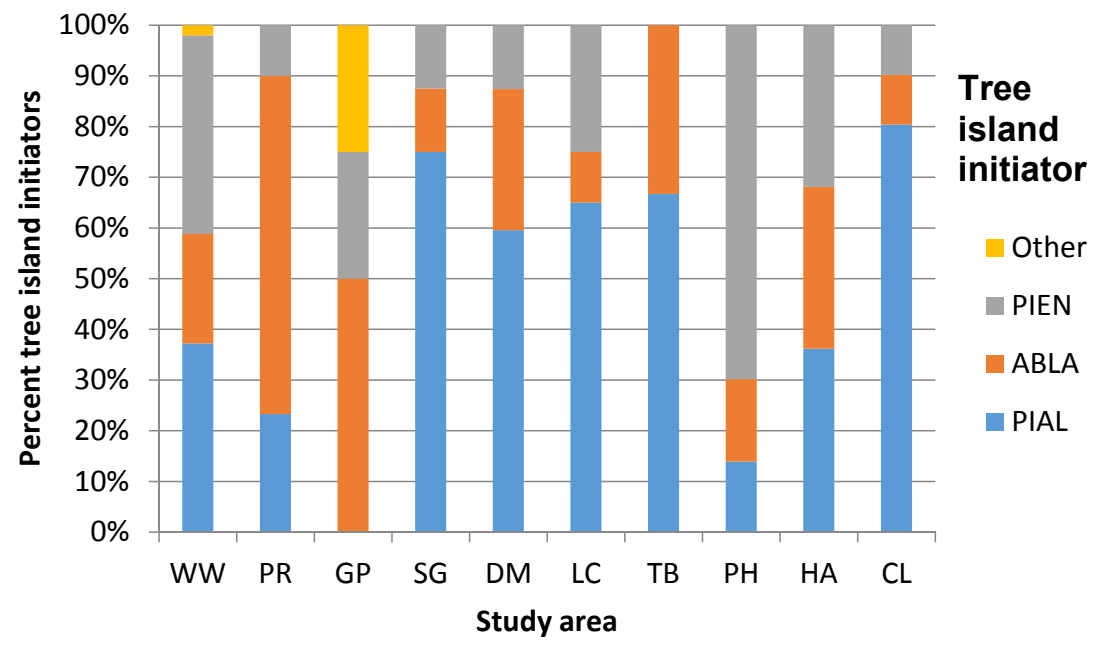

Figure 5. Relative proportional abundance of whitebark pine as a tree island initiator, as inferred by its leading windward position in tree islands, within each study area. See Table 3 for conifer species listed under "Other", and Figure 3 for conifer species abbreviations and study area abbreviations. 


\subsection{Geographic Variation in ATE Understory Community Composition}

The ATE understory vascular plant community varied among and within regions in composition (Table 3). The Willmore Wilderness Park study area understory community included lichens, especially Cladonia spp. and Stereocaulon spp. (J. Gould, personal communication), as well as large patches of multi-species heath communities (Cassiope mertensiana, Empetrum nigrum, Phyllodoce glanduliflora), which indicate substantial moisture from a maritime climatic influence [26,56]. Widely-distributed species shared among several study areas included Dryas octopetala, Arctostaphylos uva-ursi, and Silene acaulis; and, all study areas featured low-growing Salix spp., not listed in Table 3. The substrate at the Stanley Glacier and Gibbon Pass study areas comprised loose, unstable shale fragments from weathering, possibly contributing to the sparse and patchy understory at these sites.

Many of our treeline study areas are located east of the Continental Divide and exposed to the extremes of a continental climate. Exceptions include the Stanley Glacier study area, which lies west of the Continental Divide; the Divide Mountain/White Calf study area, which experiences Pacific air masses and precipitation levels similar to those west of the Divide [57]; and, the Paintbrush Divide/Holly Lake and Hurricane Pass/Avalanche Basin study areas, which occur west of the Divide within the Teton Range, a region which receives relatively heavy snowfall [56]. The latter two southern study areas included Arctostaphylos uva-ursi and Dryas octopetala in the understory community-two species characteristic of the more mesic treelines of the northern regions. The annual snowfall in the southern and eastern Wind River Range, the location of the Christina Lake study area, is half that of the Teton Range, and the region is characterized as semi-arid [56].

\subsection{Snow Retention As an Ecosystem Service}

We conducted a literature search on snowpack deposition, redistribution, and snowmelt runoff to determine whether treeline communities in general influence hydrologic processes, and whether we can extrapolate these processes to ATE whitebark pine communities. Our search revealed a long history of literature describing the spatial patterns of snow redistribution and accumulation (Table 4). These studies, which originated with the implementation of snow fences to make winter highway driving safer (e.g., [58,59]), led to the development of algorithms describing snow redistribution by wind, which indicated that objects act as snow-traps, effectively forcing snow distribution in their lee [58,60-64]. Watershed studies in arid, western plant communities indicated that strategically-placed snow fences increased snow accumulation, snowmelt discharge and the length of the flow period ([65] and references therein). Subsequent subalpine and treeline forest observational studies verified that snow depth is positively associated with tree distribution, as observed for snow fences [43,66-70] (Figure 6, Table 4). Snow depth influences other hydrological processes, including snow water equivalent (SWE), runoff initiation date, snow cover season length, and the spatial pattern of snow disappearance[41,69-72] (Table 4). Delayed runoff initiation prolongs snowdrift disappearance in areas with deeper snow [70].

Snow accumulation and distribution drive other ecosystem processes at treeline including allochthonous deposition of nutrients and herbaceous community structure [68,73-78] (Table 4). Treeline environments experience similar rates of inorganic nutrient deposition rates as subalpine forest, and higher rates than treeless alpine tundra [68]. Snowpack depth and snow cover duration also influence plant community composition and spatial distribution at both local and landscape scales [73-76], because snow-free date determines growing season length, water availability, and soil temperatures $[72,74]$. 
Table 4. References describing snowpack deposition, redistribution, snowmelt run-off, and the role of high elevation tree communities in these processes. The processes are summarized with respect to potential snow retention ecosystem services provided by whitebark pine communities in the alpine-treeline ecotone.

\begin{tabular}{ll}
\hline Topic & References \\
\hline Algorithms and highway safety research indicate wind distributes snow leeward of objects. & {$[58,60-62]$} \\
\hline $\begin{array}{l}\text { Field measurements demonstrate increased snow accumulation, } \\
\text { snow depth, and snow water equivalent leeward of trees. }\end{array}$ & {$[42,66-69,80]$} \\
\hline Increased snow depth positively correlates with snow water equivalent (SWE). & {$[69,70]$} \\
\hline Increased snow depth is associated with delayed runoff initiation and longer snow persistence. & {$[41,70-72]$} \\
\hline Snow depth and snowpack persistence predict herbaceous vegetation community distribution. & {$[73-76]$} \\
\hline Snow accumulation is positively associated with soil nutrient deposition. & {$[68,77,78]$} \\
\hline $\begin{array}{l}\text { Field based assessment of a whitebark pine ecosystem indicates } \\
\text { vegetation influences snow accumulation and spatial distribution. }\end{array}$ & {$[66]$} \\
\hline Summary of processes & \\
\hline 1. Objects, such as trees and rocks, act as snowtraps, forcing snow redistribution in their lee. & \\
\hline 2. The distribution of trees on the landscape positively influences snow depth. & \\
\hline 3. An increase in snow depth directly increases downstream water yield. & \\
\hline $\begin{array}{l}\text { 4. Whitebark pine is abundant at treeline and initiates tree islands, } \\
\text { creating snow traps, and thus influences local hydrological processes. }\end{array}$ & \\
\hline
\end{tabular}

Thus, the presence of tree stands and tree islands influences local hydrologic processes, leading to deeper snow deposition in their lee and directing the spatial distribution of snow (Figure 6). Because whitebark pine is the dominant conifer in many ATE communities throughout the Rocky Mountains and often initiates tree island development $[38,45,79]$, whitebark pine communities influence snow depth in their lee and contribute to the spatial heterogeneity of herbaceous community composition at treeline. Even if whitebark pine is not locally important in tree island initiation, it is typically an important structural component of tree islands.

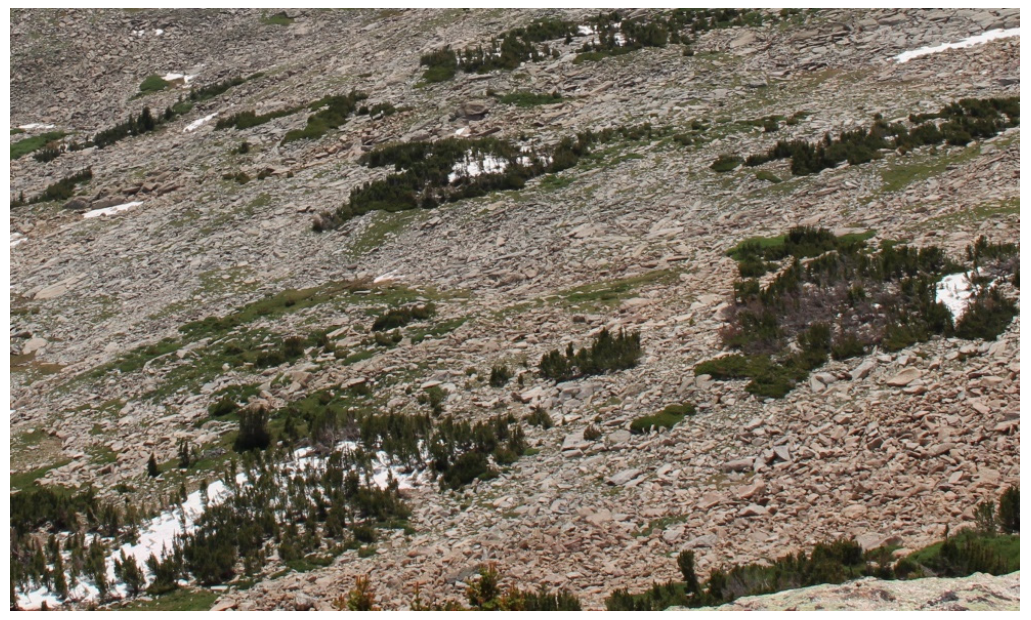

(a)

Figure 6. Cont. 


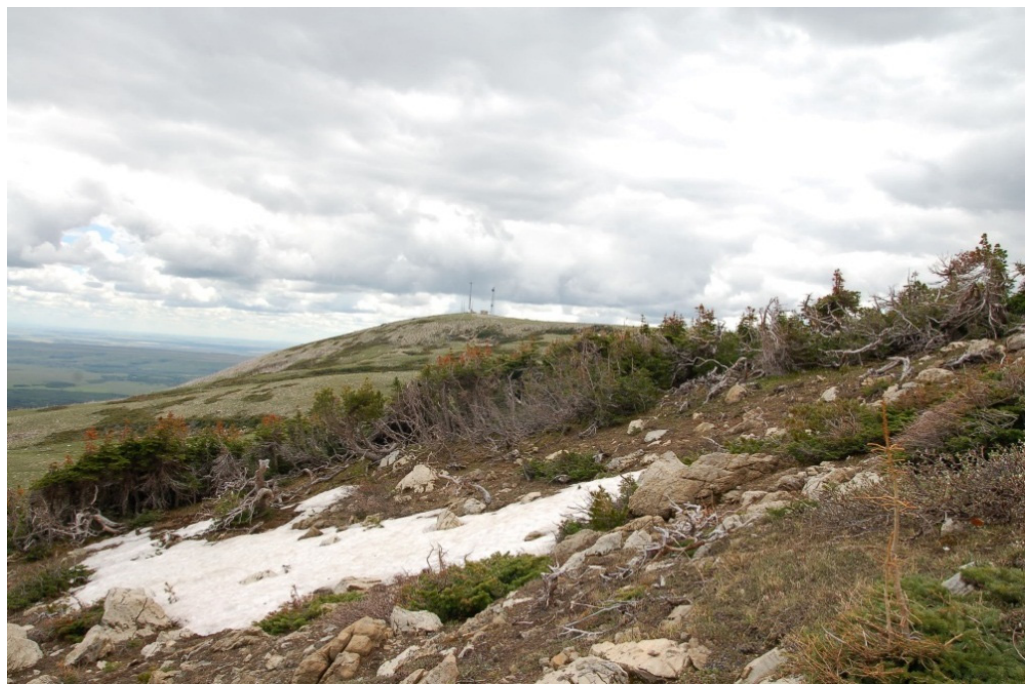

(b)

Figure 6. (a) Ribbon forest ATE community above Christina Lake, WY, with whitebark pine a major component after snowmelt completion in the surrounding treeless matrix (Photo: E.R. Pansing); (b) Whitebark pine ATE community from Divide Mountain, MT, with snowdrift in lee of tree island stringer (Photo: D.F. Tomback).

\subsection{Blister Rust Incidence Regionally and Within Treeline Communities}

White pine blister rust now occurs throughout whitebark pine's Rocky Mountain distribution in subalpine forest communities, with lower incidence at higher latitudes and east of the Continental Divide (Table 5) ([81,82], see Table 1.1). Repeated surveys on permanent plots indicate widespread infection in both the Central Rocky Mountains and Northern Rocky Mountains and tree mortality from the disease $[83,84]$. White pine blister rust is present across all regions at treeline but at low incidence at the northern and southern distributional limits, where climatic conditions are less favorable for the disease (Table 5) [85,86].

Table 5. Incidence of infection by white pine blister rust (BR) in subalpine forests in the Central and Northern Rocky Mountains, and incidence of blister rust at treeline across the Rocky Mountains.

\begin{tabular}{|c|c|c|}
\hline Location & Overall Infection Incidence; Mortality Notes & Reference \\
\hline Subalpine: Greater Yellowstone Area & $20 \%-30 \%$ mortality indicated & [83] \\
\hline Subalpine: Canadian Rocky Mountains & $52 \% 28 \%$ of trees, primarily BR & [81] \\
\hline $\begin{array}{l}\text { Treeline: east slope Glacier National Park: } \\
\text { Divide Mountain and Lee Ridge }\end{array}$ & $\begin{array}{l}33.7 \% \text { multiple symptoms; } 24.3 \% \text { confirmed } \\
\text { cankered; mortality in both study areas: } \\
6 \text { dead trees on } 3 \text { transects ; } 6 \text { dead trees } \\
\text { on } 4 \text { transects; confirmed BR }\end{array}$ & [38] \\
\hline Treeline: Willmore Wilderness Park & $1.1 \%$ (confirmed cankers) & $\begin{array}{l}\text { Tomback and Resler, } \\
\text { in preparation [51] }\end{array}$ \\
\hline Treeline: Glacier National park, 6 study areas & $47 \%(0 \%-100 \%$ per plot $)$ & {$[48]$} \\
\hline Treeline: Divide Mountain; Line Creek & $\begin{array}{l}23.6 \% \text { (confirmed cankers); } \\
19.2 \% \text { (confirmed cankers) }\end{array}$ & {$[49]$} \\
\hline Treeline: Gibbon Pass; & $0 \%$ & \\
\hline $\begin{array}{l}\text { Stanley Glacier; } \\
\text { Tibbs Butte }\end{array}$ & $\begin{array}{c}16.2 \% \text { (multiple symptoms); } \\
10.8 \% \text { (confirmed cankers); } \\
0 \% \text { (on transects; presence off transects) }\end{array}$ & [45] \\
\hline $\begin{array}{c}\text { Treeline: Paintbrush Divide/Holly Lake; } \\
\text { Hurricane Pass/Avalanche Basin }\end{array}$ & $17.2 \% ; 14.9 \%$ & $\begin{array}{l}\text { Resler and Shao } \\
\text { unpublished data [55] }\end{array}$ \\
\hline $\begin{array}{l}\text { Treeline: Tibbs Butte; } \\
\text { Christina Lake }\end{array}$ & $<10 \%$ blister rust in both areas & $\begin{array}{l}\text { Wagner et al., } \\
\text { in preparation [54] }\end{array}$ \\
\hline
\end{tabular}


The highest incidences of blister rust in subalpine whitebark pine communities occur in the Northern Continental Divide Ecosystem (e.g., Glacier and Waterton Lakes National Parks) [81], where high treeline infection levels also reflect these trends (Table 5). In the Greater Yellowstone Area, infection at the Line Creek study area was comparable to levels generally within subalpine communities (Table 5) [83]. The outlook for blister rust in North America is continued spread and regional intensification throughout the collective ranges of whitebark pine and other five-needle white pines $[15,86]$.

\section{Discussion}

\subsection{Potential Impacts to ATE Whitebark Pine Communities from White Pine Blister Rust}

Tomback and Resler [79] indicated that Cronartium ribicola could disrupt ATE communities in two ways. First, loss of seed production in subalpine whitebark pine communities from tree mortality and damage to tree canopies reduces the likelihood of seed dispersal from the subalpine communities by Clark's nutcrackers to treeline [20,87-89]. Conifers in the ATE only rarely produce seed cones, and often the seeds are not viable; the seed sources for ATE conifer regeneration are trees from lower elevations [90]. Secondly, blister rust infection at treeline damages and kills trees, potentially reducing the availability of whitebark pine to serve as a tree island initiator, which is especially important on the harshest and highest treeline sites, but also potentially eliminates whitebark pine as a frequent component of tree islands [38]. In this study, we found a direct relationship between the proportion of solitary whitebark pine and the proportion of tree islands initiated by whitebark pine, suggesting that a decline in whitebark pine could also lead to altered community composition and reduction in tree island density [79]. Understory plant composition may change as well, since patterns of snow retention and nutrient deposition may be impacted. Reduction in tree island size or density may reduce the effectiveness of snow retention in the ATE, which will further influence community composition.

Given the functional role of whitebark pine as a tree island initiator, mortality from blister rust may alter treeline response to climate change [79]. If loss of seed dispersal rrestricts the upward elevational movement of whitebark pine in response to increasing temperatures, or whitebark pine established above the limits of the current ATE succumbs to blister rust, then opportunities for tree island development may be reduced. This could lead to the perception that treeline is either slow to respond to warming trends or is not moving upwards in response to warming [79]. Agent-based modeling of the scenario "climate warming and disease" indicated that over a 500 to 800 year timeframe, whitebark pine in the presence of blister rust would decline by over $60 \%$, Engelmann spruce exceeded whitebark pine in abundance after year 630, but the area occupied by tundra increased as the area occupied by conifers declined [91].

\subsection{Geographic Variation in ATE Whitebark Pine Communities}

Although ATE whitebark pine communities are principally composed of three conifer species throughout their Rocky Mountain range, we see a difference in structure and composition among study areas within regions and across regions, primarily in response to topographic and climatic variation, but also in response to biotic factors, such as seed availability and site suitability [90]. Median density of solitary trees varies more than four-fold across study areas, and the median density of tree islands varies from 0 to 130 tree islands/ha. In different study areas, additional conifer species are part of the ATE community, and the relative species composition of both solitary trees and tree islands varies among them. Furthermore, the understory communities may share some of the same plant species, but the associations and even relative abundances vary across study areas. Thus, whitebark pine treeline communities are highly variable, and based on working definitions of biodiversity [7,92], even highly biodiverse. 


\subsection{The role of Whitebark Pine Communities In Snow Retention}

Trees within the ATE help determine snow spatial distribution across the landscape by increasing snow depth in their lee $[42,62,67,68,80]$. Snow depth affects snowpack characteristics, including SWE, timing of snowmelt initiation and runoff, and snow disappearance dates $[42,58,62,70,73,80]$. Snow distribution also influences ecosystem structure through nutrient deposition and herbaceous community composition and distribution by prolonging snowmelt initiation and snowmelt dates [68,77].

Because the presence of trees at high elevations impacts hydrologic processes, whitebark pine and whitebark pine communities potentially serve important roles in increasing snow depth locally and determining the spatial distribution of snow. Whitebark pine's tolerance of windswept, droughty sites [24,26]) combined with its role as a tree island initiator and major structural component of tree islands suggest that, in some watersheds in particular, whitebark pine not only assumes an important role in mediating hydrological processes but also influences local ecosystem processes beyond runoff dynamics, including nutrient capture and distribution, vegetation community composition, and vegetation spatial distribution (e.g., [65,68,73-78]). Although these hydrological processes are a logical extrapolation of known mechanisms, the role of treeline whitebark pine communities should be substantiated by empirical data.

\subsection{Management Implications}

Forest managers in the U.S. and Canada recognize that the growing losses of whitebark pine impact forest community biodiversity and lead to declining ecosystem function and services $[17,18]$. Restoration strategies for subalpine whitebark pine communities now exist or are under development [82,93,94]. For example, Keane et al. [82] presented general approaches for restoring whitebark pine, and Keane et al. [94] developed recommendations to mitigate climate change impacts.

We offer ideas for a two-pronged restoration strategy for ATE whitebark pine communities, which are experiencing mortality from blister rust [38]. First, existing restoration protocols apply to whitebark pine communities at the upper limits of the subalpine zone, which are the seed sources for ATE communities [87,90]. The most effective restoration treatment is to plant seedlings grown from seed sources previously identified as having some degree of genetic blister rust-resistance in areas with high tree mortality, such as after recent fires, after pine beetle outbreaks, or with high rates of blister rust [82]. This potentially increases the representation of resistance in populations, but is difficult to implement at large scales. Treatments such as thinning and prescribed burning reduce competition from faster-growing shade-tolerant trees [95]; these treatments are generally not appropriate for whitebark pine stands just below the ATE, which tend to be open, self-replacing climax communities.

Secondly, whitebark pine seedlings should be planted throughout the ATE where blister rust incidence is high to compensate for solitary krummholz tree mortality and reduced rates of seed dispersal from the upper subalpine communities. Although whitebark pine may grow without a protective microsite or nurse object in the ATE [46], survival is more likely if whitebark pine is planted leeward of a protective nurse object, such as a rock or shrub. A logistically efficient alternative to planting may be sowing seeds with potential genetic resistance to blister rust in protected sites in the ATE. Pansing et al. [96] simulated nutcracker seed dispersal by sowing caches of whitebark pine seeds in the ATE at the Tibbs Butte and White Calf Mountain study areas. Although $60 \%$ of the sown caches in both study areas were pilfered by rodents, the remaining seeds experienced greater than $60 \%$ germination in both study areas and one-year seedling survival of about $70 \%$ at Tibbs Butte and 30\% survival at White Calf. Seedlings near rocks and trees had higher survival rates than in unprotected sites. These survival rates are sufficiently reasonable to suggest that for the ATE, direct seed sowing may be more cost effective and logistically feasible than growing seedlings in nurseries for two or three years and contracting planting crews, which is the current protocol. 


\section{Conclusions}

Treeline whitebark pine communities across the Rocky Mountain distribution of whitebark are highly variable, and thus highly biodiverse, according to an inclusive definition of biodiversity [7]. Treeline communities perform the important ecosystem service of snow retention and protraction of snowmelt. Whitebark pine is a major structural component of many Rocky Mountain treeline communities, and in a subset of communities is often the principal initiator of tree islands. Loss of whitebark pine to white pine blister rust will alter treeline community composition and structure, and potentially alters response to climate warming. In addition, reduction in density of tree islands on harsh sites may reduce snow deposition and retention in these communities, altering local hydrology. We suggest that current whitebark pine restoration strategies should include planting blister rust-resistant whitebark pine seedlings at the highest subalpine elevations but also sowing seeds within the ATE at suitable sites.

Acknowledgments: We thank Joyce Gould, Alberta Parks, for information on treeline understory plant communities in Willmore Wilderness Park. The studies that provided data for this paper were funded by the National Science Foundation (grant 808548 to LMR, DFT, and G. P. Malanson); University of Colorado Denver, Faculty Development Grant (to DFT); Shoshone National Forest (grant FS-1500-17B, to DFT); and, University of Wyoming and U.S. Department of Interior, National Park Service (award ID 1002614E-VATECH to LMR). Manuscript preparation in part was supported by a Charles Bullard Harvard Forest Fellowship, Harvard University, to DFT.

Author Contributions: Diana F. Tomback, Lynn M. Resler, Robert E. Keane, and Elizabeth R. Pansing designed the study. Lynn M. Resler and Aaron C. Wagner provided unpublished data for use in the analysis; Diana F. Tomback and Andrew J. Andrade compiled data; Aaron C. Wagner and Andrew J. Andrade performed data analyses. Diana F. Tomback, Lynn M. Resler, Elizabeth R. Pansing, Robert E. Keane, and Andrew J. Andrade wrote the manuscript.

Conflicts of Interest: The authors declare no conflict of interest.

\section{References}

1. Pan, Y.; Birdsey, R.A.; Phillips, O.L.; Jackson, R.B. The structure, distribution, and biomass of the world's forests. Annu. Rev. Ecol. Syst. 2013, 44, 593-622. [CrossRef]

2. Ricketts, T.H.; Dinerstein, E.; Olson, D.M.; Loucks, C.J.; Eichbaum, W.; DellaSala, D.; Kavanagh, K.; Hedao, P.; Hurley, P.T.; Carney, K.M.; et al. Terrestrial Ecoregions of North America: A Conservation Assessment; Island Press: Washington, WA, USA; Covelo, CA, USA, 1999.

3. Habeck, J.R.; Mutch, R.W. Fire-dependent forests in the northern Rocky Mountains. Quat. Res. 1973, 3, 408-424. [CrossRef]

4. Romme, W.H.; Despain, D.G. The long history of fire in the Greater Yellowstone Ecosystem. West. Wildlands $1989,15,10-17$.

5. Fischer, W.C.; Clayton, B.D. Fire Ecology of Montana Forest Habitat types East of the Continental Divide; General Technical Report INT-141; USDA Forest Service Intermountain Forest and Range Experiment Station: Ogden, UT, USA, 1983.

6. Turner, M.G.; Hargrove, W.W.; Gardner, R.H.; Romme, W.H. Effects of fire on landscape heterogeneity in Yellowstone National Park, Wyoming. J. Veg. Sci. 1994, 5, 731-742. [CrossRef]

7. Noss, R.F. Indicators for monitoring biodiversity: A hierarchical approach. Conserv. Biol. 1990, 4, 355-364. [CrossRef]

8. Pederson, G.T.; Graumlich, L.J.; Fagre, D.B.; Kipfer, T.; Muhlfeld, C.C. A century of climate and ecosystem change in Western Montana: What do temperature trends portend? Clim. Chang. 2011, 98, 133-154. [CrossRef]

9. Westerling, A.L.; Hidalgo, H.G.; Cayan, D.R.; Swetnam, T.W. Warming and earlier spring increase western U.S. forest wildfire activity. Science 2006, 313, 940-943. [CrossRef] [PubMed]

10. Van Mantgem, P.J.; Stephenson, N.L.; Byrne, J.C.; Daniels, L.D.; Franklin, J.F.; Fulé, P.Z.; Harmon, M.E.; Larson, A.J.; Smith, J.M.; Taylor, A.H.; et al. Widespread increase of tree mortality rates in the western United States. Science 2009, 323, 521-524. [CrossRef] [PubMed] 
11. Raffa, K.F.; Aukema, B.H.; Bentz, B.J.; Carroll, A.L.; Hicke, J.A.; Turner, M.G.; Romme, W.H. Cross-scale drivers of natural disturbances prone to anthropogenic amplification: The dynamics of bark beetle eruptions. BioScience 2008, 58, 501-517. [CrossRef]

12. Weed, A.S.; Ayres, M.P.; Hicke, J.A. Consequences of climate change for biotic disturbances in North American forests. Ecol. Monogr. 2013, 83, 441-470. [CrossRef]

13. Roy, B.A.; Alexander, H.M.; Davidson, J.; Campbell, F.T.; Burdon, J.J.; Sniezko, R.; Brasier, C. Increasing forest loss worldwide from invasive pests requires new trade regulations. Front. Ecol. Environ. 2014, 12, 457-465. [CrossRef]

14. Millar, C.I.; Stephenson, N.L. Temperate forest health in an era of emerging megadisturbance. Science 2015, 349, 823-826. [CrossRef] [PubMed]

15. Tomback, D.F.; Achuff, P. Blister rust and western forest biodiversity: Ecology, values and outlook for white pines. For. Pathol. 2010, 40, 186-225. [CrossRef]

16. Tomback, D.F.; Achuff, P.; Schoettle, A.W.; Schwandt, J.; Mastrogiuseppe, R.J. The Magnificent High-Elevation Five-Needle White Pines: Ecological Roles and Future Outlook. In Proceedings High-Five Symposium: The Future of High-Elevation Five-Needle White Pines in Western North America; Keane, R.E., Tomback, D.F., Murray, M.P., Smith, C.M., Eds.; USDA Forest Service, Rocky Mountain Research Station: Fort Collins, CO, USA, 2011; pp. 2-28.

17. U.S. Fish and Wildlife Service. Endangered and threatened wildlife and plants; 12-month finding on a petition to list Pinus albicaulis as Endangered or Threatened with critical habitat. Fed. Regist. 2011, 76, 42631-42654.

18. Government of Canada. Order amending Schedule 1 to the Species at Risk Act. Canada Gazette, 2012; Part II. Vol. 146. No. 14, SOR/2012-113. Available online: http://www.sararegistry.gc.ca/ virtual_sara/files/orders/g2-14614i_e.pdf (accessed on 20 June 2012).

19. Tomback, D.F.; Linhart, Y.B. The evolution of bird-dispersed pines. Evolut. Ecol. 1990, 4, 185-219. [CrossRef]

20. Tomback, D.F. Clark's Nutcracker: Agent of regeneration. In Whitebark Pine Communities: Ecology and Restoration; Tomback, D.F., Arno, S.F., Keane, R.E., Eds.; Island Press: Washington, DC, USA, 2001; pp. 89-104.

21. Tomback, D.F. The impact of seed dispersal by Clark's nutcracker on whitebark pine: Multi-Scale perspective on a high mountain mutualism. In Mountain Ecosystems: Studies in Treeline Ecology; Broll, G., Keplin, B., Eds.; Springer: Berlin, Germany, 2005; pp. 181-201.

22. Tomback, D.F.; Anderies, A.J.; Carsey, K.S.; Powell, M.L.; Mellmann-Brown, S. Delayed seed germination in whitebark pine and regeneration patterns following the Yellowstone fires. Ecology 2001, 82, 2587-2600. [CrossRef]

23. Gernandt, D.S.; Geada López, G.G.; Ortiz Garcia, S.; Liston, A. Phylogeny and classification of Pinus. Taxon 2005, 54, 29-42. [CrossRef]

24. Arno, S.F.; Hoff, R.J. Pinus albicaulis Engelm. Whitebark pine. In Silvics of North America; Conifers; Burns, R.P., Honkala, B.H., Eds.; USDA Forest Service: Washington, DC, USA, 1990; Volume 1, pp. 268-279.

25. McCune, B. Ecological diversity in North American pines. Am. J. Bot. 1988, 75, 353-368. [CrossRef]

26. Arno, S.F. Community types and natural disturbance processes. In Whitebark Pine Communities: Ecology and Restoration; Tomback, D.F., Arno, S.F., Keane, R.E., Eds.; Island Press: Washington, WA, USA, 2001; pp. $74-88$.

27. Whitebark Pine Ecosystem Foundation. Rangewide Map for Whitebark Pine. Whitebark Pine Ecosystem Foundation: 2015. Available online: http://Whitebarkfound.org/wp-content/uploads/2014/05/ whitebark-pine-range-colour-2014.jpg (accessed on 10 August 2015).

28. Tomback, D.F.; Arno, S.F.; Keane, R.E. The compelling case for management intervention. In Whitebark Pine Communities: Ecology and Restoration; Tomback, D.F., Arno, S.F., Keane, R.E., Eds.; Island Press: Washington, DC, USA, 2001; pp. 3-25.

29. Dayton, P.K. Towards an Understanding of Community Resilience and the Potential Effects of Enrichments to the Benthos at McMurdo Sound, Antarctica. In Proceedings of the Colloquium on Conservation Problems in Antarctica; Parker, B.C., Ed.; Allen Press: Lawrence, KS, USA, 1972; pp. 81-96.

30. Ellison, A.M.; Bank, M.S.; Clinton, B.D.; Colburn, E.A.; Elliott, K.; Ford, C.R.; Foster, D.R.; Kloeppel, B.D.; Knoepp, J.D.; Lovett, G.M.; et al. Loss of foundation species: Consequences for the structure and dynamics of forested ecosystems. Front. Ecol. Environ. 2005, 3, 479-486. [CrossRef] 
31. Mills, L.S.; Soulé, M.E.; Doak, D.F. The keystone-species concept in ecology and conservation. BioScience 1993, 43, 219-224. [CrossRef]

32. Soulé, M.E.; Estes, J.A.; Berger, J.; Martinez del Rio, C. Ecological effectiveness: Conservation goals for interactive species. Conserv. Biol. 2003, 17, 1238-1250. [CrossRef]

33. Costanza, R.; d'Arge, R.; de Groot, R.; Farber, S.; Grasso, M.; Hannon, B.; Limburg, K.; Naeem, S.; O'Neill, R.V.; Paruelo, J.; et al. The value of the world's ecosystem services and natural capital. Nature 1997, 387, 253-260. [CrossRef]

34. Millenium Ecosystem Assessment. Ecosystem and Human Well-being: Synthesis; Island Press: Washington, DC, USA, 2005.

35. Farnes, P.E. SNOTEL and Snow Course Data: Describing the Hydrology of Whitebark Pine Ecosystems. In Proceedings-Symposium on Whitebark Pine Ecosystems: Ecology and Management of a High-Mountain Resource; Schmidt, W.C., McDonald, K.J., Eds.; General Technical Report INT-270; USDA Forest Service, Intermountain Research Station: Ogden, UT, USA, 1990; pp. 302-304.

36. Callaway, R.M. Competition and facilitation on elevation gradients in subalpine forests of the northern Rocky Mountains, USA. Oikos 1998, 2, 561-573. [CrossRef]

37. Tomback, D.F.; Kendall, K.C. Biodiversity losses: The downward spiral. In Whitebark Pine Communities: Ecology and Restoration; Tomback, D.F., Arno, S.F., Keane, R.E., Eds.; Island Press: Washington, DC, USA, 2001; pp. 243-262.

38. Resler, L.M.; Tomback, D.F. Blister rust prevalence in krummholz whitebark pine: Implications for treeline dynamics. Arct. Antarct. Alp. Res. 2008, 40, 161-170. [CrossRef]

39. Moerman, D.E. Native American Ethnobotany; Timber Press: Portland, OR, USA, 1998.

40. Moerman, D.E. Native American Medicinal Plants: An Ethnobotany dictionary; Timber Press: Portland, OR, USA, 2009.

41. Marsh, P.; Quinton, B.; Pomeroy, J. Hydrological Processes and Runoff at the Arctic Treeline in Northwestern Canada. In Proceedings of the Tenth International Northern Research Basins Symposium and Workshop, Norway, 1994; Sand, K., Killingtveit, A., Eds.; SINTF: Trondheim, Norway, 1995; pp. 368-397.

42. Hiemstra, C.A.; Liston, G.E.; Reiners, W.A. Snow redistribution by wind and interactions with vegetation at upper treeline in the Medicine Bow Mountains, Wyoming, USA. Arct. Antarct. Alp. Res. 2002, 34, 262-273. [CrossRef]

43. Barnett, T.P.; Adam, J.C.; Lettenmaier, D.P. Potential impacts of a warming climate on water availability in snow-dominated regions. Nature 2005, 438, 303-309. [CrossRef] [PubMed]

44. Viviroli, D.; Dürr, H.H.; Messerli, B.; Meybeck, M. Mountains of the world, water towers for humanity, typology, mapping, and global significance. Water Resour. Res. 2007, 43, W07447. [CrossRef]

45. Tomback, D.F.; Chipman, K.G.; Resler, L.M.; Smith-McKenna, E.K.; Smith, C.M. Relative abundance and functional role of whitebark pine at treeline in the northern Rocky Mountains. Arct. Antarct. Alp. Res. 2014, 46, 407-418. [CrossRef]

46. Blakeslee, S.C. Assessing Whitebark Pine Vigor and Facilitation Roles in the Alpine Treeline Ecotone; Masters of Science, University of Colorado Denver: Denver, CO, USA, 2012.

47. Pyatt, J.C.; Tomback, D.F.; Blakeslee, S.C.; Wunder, M.B.; Resler, L.M.; Boggs, L.A.; Bevency, H.D. The importance of conifers for facilitation at treeline: Comparing biophysical characteristics of leeward microsites in whitebark pine communities. Arct. Antarct. Alp. Res. 2016. in review.

48. Smith, E.K.; Resler, L.M.; Vance, E.A.; Carstensen, L.W., Jr.; Kolivras, K.N. Blister rust incidence in treeline whitebark pine, Glacier National Park, USA: Environmental and topographic influences. Arct. Antarct. Alp. Res. 2011, 43, 107-117. [CrossRef]

49. Smith-McKenna, E.K.; Resler, L.M.; Tomback, D.F.; Zhang, H.; Malanson, G.P. Topographic influences on the distribution of white pine blister rust in Pinus albicaulis treeline communities. Écoscience 2013, 20, 215-229. [CrossRef]

50. R Core Team. R: A language and environment for statistical computing. R Foundation for Statistical Computing: Vienna, Austria, 2015; Version 3.22. Available online: https:/ /www.R-project.org/ (accessed on 2 October 2015). 
51. Tomback, D.F.; Resler, L.M. Structure and composition of Rocky Mountain treeline whitebark pine communities at their northern boundary. University of Colorado Denver: Denver, CO, USA, 2015; in preparation.

52. Resler, L.M.; Shao, Y.; Tomback, D.F.; Malanson, G.P. Predicting the functional role and occurrence of whitebark pine (Pinus albicaulis) at alpine treeline: Model accuracy and variable importance. Ann. Assoc. Am. Geogr. 2014. [CrossRef]

53. Resler, L.M; Tomback, D.F.; Malanson, G.P. Treeline community structure and composition on Parker Ridge, Banff National Park. Virginia Tech: Blacksburg, VA, USA, unpublished data; 2015.

54. Wagner, A.C.; Tomback, D.F.; Pansing, E.R. Structure and composition of treeline communities at whitebark pine's (Pinus albicaulis) southern distributional limit in the Rocky Mountains. University of Colorado: Denver, CO, USA, 2015; in preparation.

55. Resler, L.M.; Shao, Y. Assessing whitebark pine treeline communities and blister rust infection in Grand Teton National Park. Virginia Tech: Blacksburg, VA, USA, unpublished data; 2015.

56. Arno, S.F.; Hammerly, R.P. Timberline: Mountain and Arctic Forest Frontiers; Timber Press: Portland, OR, USA, 1984.

57. Finklin, A. A Climate handbook for Glacier National Park—With data for Waterton Lakes National Park; General Technical Report GTR INT-204; USDA Forest Service, Intermountain Research Station: Ogden, UT, USA, 1986.

58. Mellor, M. Blowing snow. USA Cold Reg. Res. Eng. Lab. Monogr. 1965, III-A3c, 1-52.

59. Finney, E.A. Snow control on the highways. In Michigan Engineering Experimental Station, Bulletin; Michigan State College: Lansing, MI, USA, 1934; Volume 75, pp. 1-81.

60. Calkins, D.J. Simulated snowdrift patterns. USA Cold Reg. Res. Eng. Lab. Spec. Rep. 1975, 219, 1-16.

61. Kind, R.J. Snow Drifting. In Handbook of Snow; Gray, D.M., Male, D.H., Eds.; Pergamon Press: New York, NY, USA, 1981; pp. 338-358.

62. Daly, C. Snow distribution patterns in the alpine krummholz zone. Prog. Phys. Geogr. 1984, 8, 157-175. [CrossRef]

63. Allen, J.R.L. Current Ripples; North Holland Publishing Company: Amsterdam, Netherlands, 1968.

64. Kobayashi, D. Studies of snow transport in low-level drifting snow. Inst. Low Temp. Sci. Rep. 1972, 231, 1-58.

65. Sturges, D.L. Snow fencing to increase streamflow: Preliminary results. West. Snow Confer. Proc. 1986, 54, 18-28. Available online: http://www.westernsnowconference.org/sites/westernsnowconference. org/PDFs/1986Sturges.pdf (accessed on 21 December 2015).

66. Geddes, C.A.; Brown, D.G.; Fagre, D.B. Topography and vegetation as predictors of snow water equivalent across the Alpine Treeline Ecotone at Lee Ridge, Glacier National Park, Montana, USA. Arct. Alp. Res. 2005, 37, 19-205.

67. Vajda, A.; Venäläinen, A.; Hänninen, P.; Sutinen, R. Effect of vegetation on snow cover at the northern timberline: A case study in Finnish Lapland. Silva Fenn. 2006, 40, 195-207. [CrossRef]

68. Liptzin, D.; Seastedt, T. Patterns of snow, deposition, and soil nutrients at multiple spatial scales at a Rocky Mountain tree line ecotone. J. Geophys. Res. 2009, 114, G04002. [CrossRef]

69. Jonas, T.; Marty, C.; Magnusson, J. Estimating the snow water equivalent from snow depth measurements in the Swiss Alps. J. Hydrol. 2009, 378, 161-167. [CrossRef]

70. Anderton, S.P.; White, S.M.; Alvera, B. Evaluation of spatial variability in snow water equivalent for a high mountain catchment. Hydrol. Process. 2004, 19, 435-453. [CrossRef]

71. Pomeroy, J.W.; Brun, E. Physical Properties of Snow. In Snow Ecology: An Interdisciplinary Examination of Snow-Covered Ecosystems; Jones, H.G., Pomeroy, J.W., Walker, D.A., Hoham, R.W., Eds.; Cambria University Press: Cambridge, UK, 2001; pp. 45-126.

72. Tyler, S.; Burak, S.A.; McNamara, J.P.; Lamontagne, A.; Selker, J.S.; Dozier, J. Spatially distributed temperatures at the base of two mountain snowpacks measured with fiber-optic sensors. J. Glaciol. 2008, 54, 673-679. [CrossRef]

73. Billings, W.D.; Bliss, L.C. An alpine snowbank environment and its effects on vegetation, plant development, and productivity. Ecology 1959, 40, 388-397. [CrossRef] 
74. Billings, W.D. Vegetational pattern near alpine timberline as affected by fire-snowdrift interactions. Vegetatio 1969, 19, 192-207. [CrossRef]

75. Walker, D.A.; Halfpenny, J.C.; Walker, M.D.; Wessman, C.A. Long-term studies of snow-vegetation interactions. BioScience 1993, 43, 287-301. [CrossRef]

76. Odland, A.; Munkejord, H.K. Plants as indicators of snow layer duration in southern Norwegian mountains. Ecol. Indic. 2008, 8, 57-68. [CrossRef]

77. Fahnestock, J.; Povrik, K.; Welker, J. Ecological significance of litter redistribution by wind and snow in arctic landscapes. Ecography 2008, 23, 623-631. [CrossRef]

78. Nardi, A.; Bowman, W. Hot spots of inorganic nitrogen availability in an alpine-subalpine ecosystem, Colorado Front Range. Ecosystems 2011, 14, 848-863. [CrossRef]

79. Tomback, D.F.; Resler, L.M. Invasive pathogens at alpine treeline: Consequences for treeline dynamics. Phys. Geogr. 2007, 28, 397-418. [CrossRef]

80. Hiemstra, C.A.; Liston, G.E.; Reiners, W.A. Observing, modelling, and validating snow redistribution by wind in a Wyoming upper treeline landscape. Ecol. Model. 2006, 197, 35-51. [CrossRef]

81. Smith, C.M.; Wilson, B.; Rasheed, S.; Walker, R.C.; Carolin, T.; Shepherd, R. Whitebark pine and white pine blister rust in the Rocky Mountains of Canada and northern Montana. Can. J. For. Res. 2008, 38, 982-985. [CrossRef]

82. Keane, R.E.; Tomback, D.F.; Aubry, C.A.; Bower, A.D.; Campbell, E.M.; Cripps, C.L.; Jenkins, M.B.; Mahalovich, M.F.; Manning, M.; McKinney, S.T.; et al. A Range-Wide Restoration Strategy for Whitebark Pine (Pinus albicaulis); General Technical Report RMRS-GTR-279; USDA Forest Service: Fort Collins, CO, USA, 2012.

83. GYWPMWG (Greater Yellowstone Whitebark Pine Monitoring Working Group). Monitoring Whitebark Pine in the Greater Yellowstone Ecosystem; 2012 Annual Report, Natural Resource Data Series NPS/GRYN/NRDS—2013/498; Chambers, N., Ed.; U.S. Department of the Interior, National Park Service, Natural Resource Stewardship and Science: Fort Collins, CO, USA, 2013.

84. Smith, C.M.; Shepherd, B.; Gillies, C.; Stuart-Smith, J. Changes in blister rust infection and mortality in whitebark pine over time. Can. J. For. Res. 2013, 43, 90-96. [CrossRef]

85. McDonald, G.I.; Hoff, R.J. Blister rust: An introduced plague. In Whitebark Pine Communities: Ecology and Restoration; Tomback, D.F., Arno, S.F., Keane, R.E., Eds.; Island Press: Washington, DC, USA, 2001; pp. 193-220.

86. Geils, B.W.; Hummer, K.E.; Hunt, R.S. White pines, Ribes, and blister rust: A review and synthesis. For. Pathol. 2010, 40, 147-185. [CrossRef]

87. Tomback, D.F. Post-fire regeneration of krummholz whitebark pine: A consequence of nutcracker seed caching. Madroño 1986, 33, 100-110.

88. McKinney, S.T.; Fiedler, C.E.; Tomback, D.F. Invasive pathogen threatens bird-pine mutualism: Implications for sustaining a high-elevation ecosystem. Ecol. Appl. 2009, 19, 597-607. [CrossRef] [PubMed]

89. Barringer, L.; Tomback, D.F.; Wunder, M.B.; McKinney, S.T. Whitebark pine stand condition, tree abundance, and cone production as predictors of visitation by Clark's Nutcracker. PLoS ONE 2012, 7, e37663. [CrossRef] [PubMed]

90. Malanson, G.P.; Butler, D.R.; Fagre, D.B.; Walsh, S.J.; Tomback, D.F.; Daniels, L.D.; Resler, L.M.; Smith, W.K.; Weiss, D.J.; Peterson, D.L.; et al. Alpine treeline of western North America: Linking organism-to-landscape dynamics. Phys. Geogr. 2007, 28, 378-396. [CrossRef]

91. Smith-McKenna, E.K.; Malanson, G.P.; Resler, L.M.; Carstensen, L.W.; Prisley, S.P.; Tomback, D.F. Cascading effects of feedbacks, disease, and climate change on alpine treeline dynamics. Environ. Model. Softw. 2014, 62, 85-96. [CrossRef]

92. Primack, R.B. Essentials of Conservation Biology, 6th ed.; Sinauer: Sunderland, MA, USA, 2014.

93. Environment Canada. Recovery Strategy for Whitebark Pine (Pinus albicaulis) in Canada [Draft]. In Species at Risk Act Recovery Strategy Series; Environment Canada: Ottawa, ON, Canada, 2015.

94. Keane, R.E.; Holsinger, L.; Mahalovich, M.F.; Tomback, D.F. Restoring Whitebark Pine in the Face of Climate Change; General Technical Report RMRS-GTR-XXX; USDA Forest Service, Rocky Mountain Research Station: Fort Collins, CO, USA, 2016; in press. 
95. Keane, R.E.; Parsons, R. Restoring whitebark pine forests of the Northern Rocky Mountains, USA. Ecol. Restor. 2010, 28, 56-70. [CrossRef]

96. Pansing, E.R.; Tomback, D.F.; Wunder, M.B.; French, J.P.; Wagner, A.C. Simulated seed dispersal reveals geographic and community-based patterns of seed pilferage, germination, and seedling survival in whitebark pine (Pinus albicaulis). Oecologia 2016, submitted.

(c) 2016 by the authors; licensee MDPI, Basel, Switzerland. This article is an open access article distributed under the terms and conditions of the Creative Commons by Attribution (CC-BY) license (http:/ / creativecommons.org/licenses/by/4.0/). 